\title{
Assessment of quality assurance in HIV testing in health facilities in Lake Victoria zone, Tanzania
}

\author{
F.M. MASHAURI*, J.E. SIZA, M.M. TEMU, J.T. MNGARA, C. KISHAMAWE \\ and J.M. CHANGALUCHA \\ National Institute for Medical Research, Mwanza Research Centre, P.O Box 14 62, Mwanza, Tanzania
}

\begin{abstract}
Tanzania is currently implementing the antiretroviral treatment programme, and has a target of putting about 400,000 eligible HIV infected individuals on treatment by 2008. This will involve screening a large number of people, which will require non-laboratory personnel to be involved in doing HIV testing. In order to guarantee reliable and quality HIV test results, there is a need to ensure that quality assurance (QA) procedures are followed from specimen collection, testing and reporting of results. In light of the above a survey was conducted to assess QA in HIV testing in health facilities in Lake Victoria zone, Tanzania. A total of 89 health facilities (29 hospitals, 34 health centres, 9 dispensaries and 17 voluntary and counselling testing centres) were surveyed. Only three $(10.3 \%)$ health facilities reported performing Uniform II ELISA for HIV diagnosis. All other health facilities reported to be using HIV rapid tests Capillus and Determine. Five (5.6\%) of health facility laboratories performed CD4 counts. Internal quality control (IQC) were performed in $21(63.6 \%)$ of the hospitals. Kits for HIV testing were reported to be readily available by $54(60.7 \%)$ of the facilities. Only $16(18 \%)$ of the health facilities had standard operating procedures in place. Systems of equipment calibration were reported by $13(14.6 \%)$ of the health facilities. Counselling services were available in all health facilities and all counsellors had received the 6-week mandatory training course. These findings show that most of health facilities in the Lake Victoria zone do not adhere to QA procedures in HIV testing. There is therefore, a need to establish a monitoring system to laboratories performing HIV testing for the purpose of ensuring QA procedures are done. Personnel doing HIV testing should be re-trained at a regular basis to cope with new techniques and ensure QA procedures are followed.
\end{abstract}

Key words: quality assurance, health facilities, laboratory, HIV / AIDS, Tanzania

\section{Introduction}

Tanzania is currently implementing the antiretroviral treatment (ART) programme and has a target of putting 400,000 eligible HIV infected individuals on treatment by $2008(\mathrm{MoH}$, 2005). The programme entry point involves voluntary counselling and testing (VCT), prevention of mother to child HIV transmission (PMTCT), sexually transmitted infection and tuberculosis clinics. This will require screening a massive number of people, which will require non-laboratory personnel to be involved in doing HIV testing.

Early diagnosis of HIV / AIDS has been found to be the best and effective strategy for facilitating behavioural changes as well as preventing HIV transmission and allows early access to treatment and care (Marks et al., 1999). Knowing ones HIV status provides chances for someone who is negative to remain negative, and those positive to seek treatment including ART thus reducing the possibilities of transmitting the infection to their partners and children (UNFPA, 2002). Likewise, improved access to PMTCT services increases the uptake of VCT services (de Goei, 2003). Therefore, laboratory services are vital in all areas of HIV / AIDS diagnosis and management. National efforts to rapidly scale up treatment and care services in Tanzania especially the provision of ART is exclusively dependent on efficient laboratory services.

While rapid HIV tests are the mainstay of HIV testing in primary healthcare facilities and VCT centres, laboratory services in resource limited settings are generally inadequate to meet the needs of targeted population (WHO, 2003). Lack of financial investment, insufficient infrastructure, and overwhelmed personnel coupled with little awareness of quality assurance (QA) procedures makes it difficult for health facilities to maintain the quality of the HIV testing that is needed to target prevention and treatment. This is particularly important at the primary healthcare facilities where most people seek treatment and support (CDC \& WHO/ AFRO, 2001).

With the spread of HIV / AIDS particularly in sub-Saharan Africa (UNAIDS, 2004), there is an urgent need to strengthen laboratory services in order to cope with enormous workload. Demands of quality and reliable HIV test results can be met through commitment to QA by enhancement of personnel performing HIV testing. Quality 
assurance requires a logistic system that provides adequate and continual supply of HIV kits, equipment and storage facilities for blood/serum specimens. Quality assurance activities need to be performed from the time an individual consents to be tested to the time HIV test results are given and recorded (CDHSOA, 2003).

To address these urgent needs and constraints, we conducted a survey to assess the situation of QA in HIV testing in health facilities in Lake Victoria zone, Tanzania with the view to provide evidence based information in order to ensure that HIV testing is of high quality and reliable in all health facilities. The findings of this study will be useful to policy makers, programme managers and other stakeholders in looking for a feasible means to improve the quality of HIV laboratory testing in the country.

\section{Materials and Methods}

\section{Study area}

The survey was undertaken in four regions of Mwanza, Kagera, Shinyanga and Mara. Mwanza region is made up of 8 administrative districts namely Nyamagana, Ilemela, Ukerewe, Sengerema, Misungwi, Kwimba, Magu and Geita. Kagera has 7 districts that are Bukoba urban, Bukoba rural, Muleba, Ngara, Karagwe, Biharamulo and Chato. Shinyanga has 8 administrative districts, Shinyanga urban, Shinyanga rural, Bariadi, Bukombe, Kahama, Maswa, Kishapu and Meatu. Mara is made up of 5 districts, Musoma urban, Musoma rural, Bunda, Tarime and Serengeti. According to the 2002 National Census the Lake Victoria zone had a population of 9,150,218 with an intercensual growth rate of about 3\% (URT, 2003). At most 4 randomly selected health facilities providing VCT services and those performing HIV tests for other purposes like blood screening and PMTCT in 27 districts in the zone were included in the survey.

\section{Study design and data collection}

This was a cross-sectional quantitative descriptive study health facility based where structured questionnaire was used to elicit information on QA procedures in HIV testing in the study area. During the survey laboratory personnel and counsellors were interviewed using a structured questionnaire regarding types of kits used, HIV testing guidelines followed, source of kits and storage facilities for blood/serum specimens, presence of standard operating procedures (SOPs) and system of equipment calibration. Information was also collected about CD4 count, quality control procedures, level of training of staff and availability of testing kits.

\section{Data analysis}

Data were double entered, verified and cleaned using Dbase Version 4 (Borland Corporation, Scotts Valley, CA, USA). Cleaned dataset was transformed into STATA file. Calculations of proportions for health facilities visited, type of HIV tests used and QA procedures were calculated using STATA Version 9 (STATA Corporation, College Station, Texas, USA).

\section{Results}

The survey involved 89 health facilities, including $29(32.6 \%)$ hospitals, $34(38.2 \%)$ health centres, 9 $(10.1 \%)$ dispensaries and 17 (16.9\%) VCT centres (Table 1). Three health facility laboratories were using Uniform II ELISA tests for HIV testing, while the rest were using rapid tests, Capillus and Determine. Uniform II ELISA and Enzygnost tests were performed by the National Institute for Medical Research (NIMR) at its Mwanza laboratory and 2 hospitals as a confirmatory test (Table 2). In case of indeterminant or negative results HIV re-testing after 3 months was reported to be done in 21 (95\%) health facilities in Kagera, $3(17 \%)$ in Mara, $12(43 \%)$ in Mwanza and $2(8 \%)$ in Shinyanga. Three health facilities reported using WHO guidelines, 44 and 42 were following Ministry of Health and National AIDS Control programme (NACP) guidelines, respectively. The mean HIV tests per month were reported to be 100 in Kagera, 127 in Mara, 136 in Mwanza and 98 in Shinyanga. 
Table 1: The distribution of surveyed health facilities in Lake Victoria zone

\begin{tabular}{|c|c|c|c|c|c|}
\hline Facility & Kagera & Mara & Mwanza & Shinyanga & Total \\
\hline Hospital & 9 & 5 & 9 & 6 & 29 \\
\hline Health centre & 4 & 10 & 11 & 7 & 34 \\
\hline Dispensary & 6 & 1 & 0 & 4 & 9 \\
\hline VCT & 3 & 2 & 8 & 4 & 17 \\
\hline Total & 22 & 18 & 28 & 21 & 89 \\
\hline
\end{tabular}

Table 2: Type of tests used for HIV testing at health facilities levels in Lake Victoria zone

\begin{tabular}{lcccc}
\hline Test & Hospital & Health Centre & Dispensary & VCT/Laboratory $^{*}$ \\
\hline Uniform II ELISA & $2(6.9)$ & $0(0.0)$ & $0(0.0)$ & $1(5.9)$ \\
Enzygnost & $2(6.9)$ & $0(0.0)$ & $0(0.0)$ & $1(5.9)$ \\
Capillus & $29(100)$ & $34(100)$ & $9(100)$ & $17(100)$ \\
Determine & $28(96.6)$ & $32(94.1)$ & $9(100)$ & $17(100)$ \\
\hline
\end{tabular}

*NIMR Mwanza laboratory performed both Uniform II ELISA and Enzygnost

CD4 count was done at regional hospitals and NIMR Mwanza laboratory

Most of the internal quality control (IQC) procedures were performed at hospital level. Internal and external quality controls were not common $(37 \%)$ in the rest of the health facilities. SOPs were used in $16(18 \%)$ of the health facilities surveyed (Table 3). Refrigerators for storage of HIV kits and blood/serum specimens were available in $57(64 \%)$ of the health facilities, but daily temperature recording charts were uncommon. Systems of equipment calibration were reported by $13(14.6 \%)$ of the facilities.
Half of facilities used register books to record their test results. Kits for HIV testing were reported to be readily available by $54(60.7 \%)$ once required while the rest reported delays. The major supplier of the HIV test kits to health facilities was the Medical Stores Department. Other sources included NACP, Projects or Programmes and Pharmacies. Marker pens and pencils/pens were major tools for labelling specimen containers. Very few $6(7 \%)$ facilities reported using stickers.

Table 3: Quality assurance activities performed in HIV testing at health facilities levels in Lake zone

\begin{tabular}{lrrrr}
\hline Quality assurance activity & Hospital & Health Centre & Dispensary & VCT/Lab \\
\hline IQC system & $21(63.6)$ & $4(11.8)$ & $2(22.2)$ & $7(41.2)$ \\
EQC system & $5(15.2)$ & $7(20.6)$ & $3(33.3)$ & $7(41.2)$ \\
SOPs in place & $4(12.1)$ & $3(8.9)$ & $3(33.3)$ & $6(35.3)$ \\
Daily temp recording chart & $10(40.0)$ & $5(29.4)$ & $2(33.3)$ & $8(47.1)$ \\
System of equipment calibration & $6(18.2)$ & $1(2.9)$ & $2(22.2)$ & $5(29.4)$ \\
Test results forms & $19(57.6)$ & $14(41.2)$ & $8(88.9)$ & $8(47.1)$ \\
Mechanism of accepting or rejecting & $5(15.2)$ & $2(5.9)$ & $0(0.0)$ & $1(5.9)$ \\
specimens & & & & \\
Supplies available once required & $19(57.6)$ & $18(52.9)$ & & $11(64.7)$ \\
\hline
\end{tabular}


In the aspect of training $84(94.4 \%)$ of staff doing HIV testing had received training for a duration ranging from 6 weeks to three months. Five health facilities in the zone performed CD4 counting in their laboratories. These included four regional hospitals and the NIMR Mwanza laboratory. Methods used for CD4 counting and numbers of laboratories in bracket included Flow Cytometry (1), Coulter manual (1) and Facscount method (3). Counselling services were available in all health facilities and all counsellors had received the 6week mandatory training course.

\section{Discussion}

The current National HIV rapid test algorithm recommends health facilities to carry out HIV tests using Capillus and if positive to confirm with Determine. For indeterminant or negative results, blood samples are re-tested after three months or subjected to a confirmatory test Uniform II ELISA. Capillus is used first is because it has higher sensitivity but low specificity while Determine has higher specificity but low sensitivity. Moreover, these rapid tests are cheap, easy to perform and results are obtained within a short time as compared to ELISA test (Stetler, 1997). However, one health facility reported to be using Determine first in HIV testing. The only problem of using Capillus HIV rapid test in rural health facilities is lack of cold storage appliance. This is of particular concerns for rural health facilities which do not have reliable source of electricity and refrigerators. There was lacks of uniformity on HIV re-testing after 3 months in case of indeterminant or negative results due to lack of SOPs. This can result into different procedures being followed by different individuals and facilities and could compromise the quality of results.

The National Quality Control Assessment Scheme (NQCAS) guideline recommends every tenth and all indeterminant samples from primary health facilities to be sent to the National HIV reference laboratories at Muhimbili National Hospital (Dar es Salaam), Bugando Medical Centre (Mwanza), Kilimanjaro Christian Medical Centre (Moshi), or Mbeya Referral Hospital (Mbeya) for external quality control. For internal quality control it is recommended to run a known positive and negative control samples daily before routine HIV testing by a laboratory personnel.

Few health facilities reported doing both internal and external quality controls, particularly at primary health facilities suggesting that the integrity and quality of results produced is questionable. For health facilities with refrigerators the absence of temperature recording charts suggest that the integrity of stored HIV kits and samples was doubtful and could lead to wrong results. Lack of system for equipment calibration was reported by the majority of the surveyed facilities. This suggests that precision and accuracy of measurements are uncertain, and this may affect the quality of results produced. Half of facilities used register books to record their test results instead of forms probably due to the costs involved in recurrent procurement of stationery. However some health facilities reported the tedious work of keeping separate forms together as compared to an already compact book mainly because they were not giving written results to their clients. The availability and use of both WHO, Ministry of Health and NACP guidelines in three healthcare facilities were unexpected. The NACP belongs to the Ministry of Health, thus there was no justifiable reasons for having separate guidelines.

The major supplier of the HIV test kits was reported to be MSD. However, there was a great concern regarding the delay of supply of the kits and in some circumstances only one type HIV test kit was supplied. CD4 quantification was done only at the regional hospitals which means that the primary health facilities have to transport blood specimen to the regional hospitals. This process may results into delay of initiation of ART and may hinders monitoring and evaluation of treatment outcomes. Provision of CD4 count machine to district hospitals will allow individuals eligible for ARV provision to access the service easily.

In conclusion, these findings show that most of health facilities in the Lake Victoria zone do not adhere to QA procedures in HIV testing. Non adherence to QA means poor quality and unreliable HIV test results. In this regard there is a need to establish a monitoring system to laboratories performing HIV testing for the purpose ensuring that activities related to QA procedures are followed. Personnel doing HIV 
testing should be re-trained on the procedures of HIV testing and quality assurance. Lack of CD4 count machines at district hospitals reflects that majority of poor people living in rural settings may be left out in the national ART scaling up programme. The government should therefore, provide the machines to all the public healthcare facilities.

\section{Acknowledgements}

We are very grateful to all people and organizations that enabled this survey a success. Sincere appreciations are also extended to Regional Medical Officers of Kagera, Mara, Mwanza and Shinyanga and District Medical Officers for their tireless cooperation during the survey. We are greatly indebted to all laboratory personnel and counsellors in the visited health facilities for their patience during the interview. This study received financial support from the National Institute for Medical Research through the HIV / AIDS Care and Treatment Programme.

\section{References}

CDC \& WHO/AFRO (2001) Guidelines for Appropriate Evaluation of HIV testing Technologies in Africa. pp 1-48

CDHSOA (2003) OralQuick Rapid HIV Testing Guidelines: Policies, Procedures and Quality Assurance. pp 1-98. de Goei,T. (2003) Community Preparedness for Antiretroviral Treatment. Sexual Health Exchange 2003/3

Marks, G., Burris, S. \& Peterman, T.A. (1999) Reducing sexual transmission of HIV from those who know they are infected: the need for personal and collective responsibility. AIDS 13, 297-306.

$\mathrm{MoH}$ (2005) HIV/AIDS Care and Treatment Plan. Ministry of Health, United Republic of Tanzania, Dar es Salaam.

Stetler, H.C., Granade, T.C. \& Nunez, C.A. (1997) Field evaluation of rapid HIV serologic tests for screening and confirming HIV1 infection in Honduras. AIDS 11, 369375.

UNFPA (2004) Prevention of HIV infection: promoting reproductive health: Available at http: / / www.unfpa.org/aids.htm accessed in 12/2/2004.

UNAIDS (2004) Report on the Global Burden of HIV/AIDS Epidemic. United Nations Programme on HIV / AIDS. Joint United Nations Programme on HIV / AIDS, Geneva, Switzerland. Pp19-30.

URT (2003) 2002 Population and Housing Census General Report. Central Census Office. National Bureau of Statistics, President's Office, Planning and Privatization. Government Printers. United Republic of Tanzania, $203 \mathrm{pp}$.

WHO (2003) HIV Testing and Counselling: The Gateway to Treatment, Care and Support. World Health Organization, Geneva. 